\title{
Oceanic heterotrophic flagellates are dominated by a few widespread taxa
}

\author{
Aleix Obiol ${ }^{(D,}{ }^{*}$ Imer Muhovic, Ramon Massana $\mathbb{1}^{*}$ \\ Department of Marine Biology and Oceanography, Institut de Ciències del Mar (ICM-CSIC), Barcelona, Spain
}

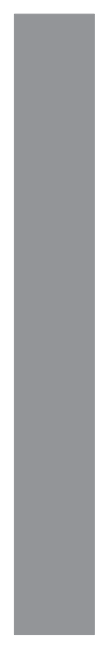

\begin{abstract}
Marine heterotrophic flagellates (HFs) form a diverse and ecologically relevant functional group of bacterial grazers and nutrient remineralizers in oceanic waters. Despite playing a crucial role in marine biogeochemical cycles, there is still a lack of information on which specific taxa dominate HF assemblages and what are their patterns of distribution in a global context. In the present work, we addressed this issue by analyzing amplicon sequencing data sets retrieved from samples taken in tropical and subtropical oceanic regions at depths from surface to $4000 \mathrm{~m}$. Only a few dozens of widespread taxa, mostly affiliating to MAST clades, Picozoa, Bicosoecida and Chrysophyceae, seemed to dominate surface HF assemblages. The majority of these dominant HFs were present at relatively constant abundances, while others were influenced by temperature or displayed a patchy distribution. In the deep ocean, only a handful of taxa belonging to Bicosoecida and Chrysophyceae, together with Diplonemea and Kinetoplastida, explained most of the HF signal. Co-occurrence networks between HF and prokaryotic taxa at the surface ocean revealed two main clusters influenced by temperature that did not seem to show specific patterns of interaction. However, some correlations emerged outside these thermal groups that could represent new prey-predator interactions. Overall, we identified the putatively most ecologically relevant HF taxa in the ocean, which become promising targets for further experimental and genomic studies.
\end{abstract}

Marine heterotrophic flagellates (HFs) are minute unpigmented eukaryotes (2-20 $\mu \mathrm{m}$ in size) that are found in the plankton at concentrations of $10^{2}-10^{4}$ cells $\mathrm{mL}^{-1}$. They represent around $20 \%$ of total eukaryotic organisms in the photic zone of the oceans (Jürgens and Massana 2008). Collectively, they form a highly diverse assemblage, with species affiliated with all major eukaryotic supergroups (Jeuck and Arndt 2013; Adl et al. 2019; Schön et al. 2021). Consideration of their importance in ocean ecosystems changed dramatically about $40 \mathrm{yr}$ ago, when it was shown that these microorganisms were active bacterial grazers and were part of marine food webs (Azam et al. 1983). Further studies confirmed that HFs, and particularly those in the 2-5 $\mu \mathrm{m}$ size range, were major agents of prokaryotic mortality in planktonic systems (Fenchel 1986), along with viruses (Fuhrman and Noble 1995), and mixotrophic protists (Zubkov and Tarran 2008). In these planktonic systems, HFs are crucial in channeling carbon to higher trophic levels and in regenerating inorganic nutrients such as nitrogen and phosphorus

*Correspondence: obiol@icm.csic.es (A. O.); ramonm@icm.csic.es (R. M.)

This is an open access article under the terms of the Creative Commons Attribution License, which permits use, distribution and reproduction in any medium, provided the original work is properly cited.

Additional Supporting Information may be found in the online version of this article. that would otherwise be kept within bacterial biomass (Sherr and Sherr 2002; Pernthaler 2005; Jürgens and Massana 2008).

Despite their significance in the environment, studying HFs presents some challenges that have impeded obtaining a detailed image of the diversity and function of natural HF assemblages. First, contrary to larger eukaryotic microorganisms, many HF cells lack conspicuous morphological traits that could be used for taxonomy, thus hindering their identification through light microscopy. Second, the available cultured strains do not generally represent the dominant species in the environment, many of which still remain uncultured (del Campo et al. 2013). So, ecophysiological studies on cultured strains (functional and numerical responses, recycling capacity, environmental responses) may poorly account for in situ ecological performances. In fact, HF assemblages have been usually treated as a black box in terms of their ecological activity in marine ecosystems, ignoring they are formed by species with distinct ecophysiologies. Molecular diversity studies have been fundamental to open this black box.

The first molecular diversity surveys of marine protists, including HFs, cloned and sequenced the $18 \mathrm{~S}$ ribosomal DNA gene as a phylogenetic marker (Diez et al. 2001; López-García et al. 2001; Moon-Van Der Staay et al. 2001; Edgcomb et al. 2002). These revealed a large taxonomic diversity in natural assemblages and allowed new uncultured lineages to be described (Massana et al. 2004; Not et al. 2007; Guillou 
et al. 2008). Recent global oceanic expeditions, using highthroughput sequencing, also surveyed eukaryotic diversity (de Vargas et al. 2015; Giner et al. 2020; Obiol et al. 2020), expanding the initial picture and creating precious resources for further detailed studies. Recent publications have used the released data to assess the diversity and biogeography of specific groups, such as diatoms (Malviya et al. 2016), dinoflagellates (Le Bescot et al. 2016), green algae (Lopes dos Santos et al. 2017; Metz et al. 2019), kinetoplastids (Flegontova et al. 2018), and ciliates (Canals et al. 2020). An equivalent study targeting the taxonomically heterogeneous HF assemblages in the water column of the oceans is still missing.

Here, we used previously released sequencing data sets to address a series of crucial questions related to understanding marine HFs at a large scale: (1) Which are the main HF taxonomic groups in marine waters? (2) Are HF assemblages shaped by environmental factors? (3) Are there globally dominant HF species and what are their spatial distributions? (4) Can we detect specific co-occurrence patterns among dominant HF species and prokaryotic taxa? To answer these questions, we analyzed V4 18S rDNA sequences assigned to HF taxonomic groups in 279 metabarcoding samples. These corresponded to the picoeukaryotic fraction $(0.2-3 \mu \mathrm{m})$ and were collected during the Malaspina 2010 circumglobal expedition in surface waters (Logares et al. 2020) and down the water column (Giner et al. 2020). We also analyzed other oceanic datasets (V9 amplicons and metagenomes) to provide additional support to the emerging view. Our results revealed a large taxonomic diversity in HF marine assemblages, a contrasted community structure in different depth zones, and the existence of a few dominant and widespread taxa that may be ecologically relevant models whose ecological roles should be examined in further studies. We also highlighted cooccurrence patterns that could represent undescribed and promising prey-predator interactions. Overall, our findings put HFs back in the spotlight in which they were placed decades ago.

\section{Materials and methods}

\section{Samples for sequencing and amplicon processing}

Samples were collected in tropical and subtropical oceans during the Malaspina 2010 Circumnavigation Expedition (Supporting Information Fig. S1, Table S1). Seawater sampling, filtration to keep the pico-sized fraction $(0.2-3 \mu \mathrm{m})$, and nucleic acids extractions are explained in the original publications for surface samples (Logares et al. 2020) and for vertical profiles (Giner et al. 2020). Here we considered 122 nucleic acid extracts from surface waters ( $3 \mathrm{~m}$ depth; DNA extracts only) and 179 from vertical profiles (88 DNA and 91 RNA extracts). The vertical profiles were collected at 13 stations sampled at surface, deep chlorophyll maximum (DCM) and 2-3 depths at mesopelagic (200-1000 m) and bathypelagic (1000-4000 m) zones. For the obtained extracts, the V4 region of the $18 \mathrm{~S}$ rDNA
( 380 bp) was amplified by Polymerase Chain Reaction (PCR) using eukaryotic universal primers (Stoeck et al. 2010) and sequenced with the MiSeq platform $(2 \times 250 \mathrm{bp})$.

We trimmed Illumina raw reads to remove amplification primers using cutadapt v1.16 (Martin 2011) and processed them with DADA2 v1.12.1 (Callahan et al. 2016). We set DADA2's truncLen parameters to 240,210 for the surface data set and 210,190 for both DNA and RNA data sets from the vertical profiles, as these were processed in separate batches and presented different quality profiles. In the three runs, we used values 6,8 for maxEE and pool=TRUE. We merged the obtained tables based on the amplicon sequence variants (ASVs) delineated. We removed chimeric ASVs with the method pooled as implemented in DADA2's function removeBimeraDenovo, and removed ASVs shorter than $300 \mathrm{bp}$ or not present in at least 2 samples. Then, we discarded 14 samples with less than 8000 reads. We added group level taxonomy (in general a formal Class) by comparing ASVs by BLAST (Altschul et al. 1990) to the eukaryotesV 4 version 4 database (Obiol et al. 2020), based in the taxonomic outline of Adl et al. (2019). When an ASV was $>95 \%$ identical in $>300$ bp alignment to an eukaryotes $V 4$ reference sequence, we assigned the taxonomic group of the reference to the ASV. When identity was between 90\% and 95\%, we manually inspected ASVs in phylogenetic trees performed with RAxML-NG (Kozlov et al. 2019) using all ASVs from the same supergroup. For identities lower than 90\%, we placed ASVs as incertae sedis (InSed). A total of 426 ASVs (0.7\% of the reads) remained unclassified even at the supergroup level and some could potentially represent very distinctive novel HF taxa. Yet, we did not consider them, as we lacked any information on their cell identity. We removed ASVs assigned to Metazoa, Charophyta, Embryophyta, and nucleomorphs $(5.3 \%$ of the reads). The final protist table contained 16,629 ASVs and 287 samples (116 from the surface data set, 80 from DNA vertical profiles, and 91 from RNA vertical profiles), with an average of 91,561 (70,064 standard deviation [SD]) reads per sample.

\section{Creating a catalogue of oceanic HFs}

We created a subset table of HFs by keeping ASVs from taxonomic groups possibly including HF morphotypes (listed in Supporting Information Table S2). We did not consider dinoflagellates, as their minimal size is about $5 \mu \mathrm{m}$, which is larger than the size fraction analyzed here. We placed the selected ASVs into previously published phylogenetic trees (Supporting Information Fig. S2) for the following groups: Bicosoecida (del Campo and Massana 2011), Centrohelida (Shishkin et al. 2018), Cercozoa (Bass et al. 2018), Choanoflagellata (del Campo and Massana 2011), Chrysophyceae (del Campo and Massana 2011), Dictyochophyceae (Sekiguchi et al. 2002), Marine Stramenopiles (MAST; Massana et al. 2014), Picozoa (Moreira and López-García 2014), and Telonemia (ShalchianTabrizi et al. 2007). For each group, we retrieved complete $18 \mathrm{~S}$ rDNA sequences using the NCBI accession numbers shown in the trees, aligned them using mafft v7.402 (Katoh and 
Standley 2013), added the ASV sequences to the alignment with mafft, and constructed maximum likelihood trees with RAxML-NG (Kozlov et al. 2019). Group-specific phylogenetic trees served to refine taxonomic placements and to identify ASVs close to plastidic species in groups encompassing colorless and pigmented taxa. Thus, we removed 137 Dictyochophyceae and 7 Cercozoa ASVs that were $>97 \%$ identical to species known to have chloroplasts, while we did not detect any ASV closely related to a plastidic Chrysophyceae. We also visually inspected the alignments and removed 133 ASVs that were obvious chimeras or partial sequences.

We filtered the obtained preliminary HF ASV table to keep samples with more than 500 reads (we removed 8 samples), and ASVs that were present in at least 2 samples. The final HF table contained 1642 ASVs from 25 taxonomic groups, 279 samples (115 from surface, 73 from DNA vertical profiles, and 91 from RNA vertical profiles), and an average of 23,906 (34,319 SD) reads per sample. With this table, we created a catalogue of oceanic HF ASVs (Supporting Information Table S3). We kept RNA samples ("V4 RNA") only for comparison purposes and used the DNA data set ("V4 DNA"), which contained 188 samples from both surface and vertical profiles, for the rest of the analyses (Supporting Information Table S1). For each DNA ASV, we performed a BLAST search against NCBI nt database excluding environmental sequences to find its closest cultured match (CCM) and added a name to its unique identifier. If identity to the CCM was $>97 \%$, we used species name and percentage of identity; otherwise, we used group name and "sp" followed by a rank abundance index. For example, the first Chrysophyceae ASV in terms of overall abundance without a close CCM was named "Chrysophyceae-sp1."

\section{A web application to map global microbial biogeographies}

We developed an online visualization tool for marine microbial biogeography. MicroMap is a web application that provides an interface for submitting queries to an ASV database and creating global maps of ASV abundances. MicroMap incorporates the $18 \mathrm{~S}$ rDNA protist database used here and the $16 \mathrm{~S}$ rDNA surface data set published in Logares et al. (2020). In addition, MicroMap can incorporate a custom-made database constructed from the users' own ASV table and sample table. Queries can be done with $16 \mathrm{~S} / 18 \mathrm{~S}$ rDNA sequences, with named taxonomic groups, or with known sequence IDs from the database. In the query with a DNA sequence, the application runs a BLAST search using the parameters configured in the home page. It takes the matching ASVs and collates them into a results file used to interactively display relative abundances in four water zones and abundance spectra plots. In queries based on taxonomic names, the application looks for ASVs classified with that name and treats all of them as a single result. In queries based on a sequence ID, the tool displays the results of that ASV. MicroMap is implemented in Laravel 5.8 using PHP 7.2, and the front end of the application runs in VueJS, with $\mathrm{R}$ scripts providing additional functionality for creating plots. The interactive map uses the Datamaps library implemented in the D3 framework. It is hosted at the ICM-CSIC on a Dell server, with 320 GB of RAM and two 2 Intel Xeon E5-2650v4 processors, and is accessible at https:// micromap.icm.csic.es/.

\section{Other data sets used for comparison and co-occurrence analyses}

We analyzed two additional molecular data sets to support the HF diversity and distribution results obtained by 18S-V4 amplicons (Supporting Information Table S1): (1) 70 V9-18S rDNA amplicon sequencing samples $(0.8-5 \mu \mathrm{m}$ size fraction; "TARA V9") from surface and DCM waters from TARA Oceans (de Vargas et al. 2015; Callahan 2017), and (2) 66 metagenomes ("mTags") and 34 V9-18S rDNA amplicon sequencing samples ("V9 DNA") from vertical profiles from the pico fraction (0.2$3 \mu \mathrm{m}$ ) from Malaspina (Obiol et al. 2020). For co-occurrence analyses with prokaryotes, we used the V4-V5 16S rDNA data set ("Prokaryotes") from Logares et al. (2020), which comprised 115 surface samples from the same DNA extracts as the $18 \mathrm{~S}$ rDNA data set. All samples used are listed in Supporting Information Table S1.

\section{Statistical analyses}

We performed general processing of ASV tables using $\mathrm{R}$ v4.0.5 (R Core Team 2020) and packages tidyverse v1.3.1 (Wickham et al. 2019) and phyloseq v1.34.0 (McMurdie and Holmes 2013). We conducted principal coordinate analysis (PCoA), non-metric multidimensional scaling (NMDS), and permutational multivariate analysis of variance (PERMANOVA) with vegan v2.5.7 (Oksanen et al. 2019) using BrayCurtis dissimilarity matrices computed with the package DivNet v0.3.6 (Willis and Martin 2020). We performed differential abundance tests of HF and prokaryotic ASVs with scaled environmental variables with the package corncob v0.1.0 (Martin et al. 2020). To describe the distribution pattern of surface HF ASVs, we used the ratio $\mathrm{SD} /$ mean of the relative abundance over samples; an ASV was considered "patchy" when this was higher than 2.3. When the ratio was lower, an ASV was considered "warm" or "cold" if it had a significant estimate across temperature in the corncob test (estimate $>0.5$, $p<0.01)$ and "equal" when the test was not significant. We carried out co-occurrence network analyses using sparCC (Friedman and Alm 2012) as implemented in fastSpar v0.0.7 (Watts et al. 2019). We used EnDED (Deutschmann et al. 2019) as implemented in Deutschmann et al. (2021) on the obtained correlation matrix to remove associations driven by environmental variables (temperature, conductivity, dissolved oxygen, and fluorescence). We carried out network processing with tidygraph v1.2.0 (Pedersen 2020a) and ggraph v2.0.5 (Pedersen 2020b). We performed DivNet, corncob, and sparCC analyses on absolute counts tables, as these packages 
already take into consideration the compositional nature of microbiome data (Gloor et al. 2017).

\section{Results}

General overview of HF assemblages in the ocean

The processing of V4 $18 \mathrm{~S}$ rDNA reads derived from DNA extracts taken at the surface and from vertical profiles in Malaspina (Supporting Information Fig. S1) yielded 1030 ASVs representing HF taxa within 24 taxonomic groups (Supporting Information Table S3, Fig. S2). The contribution of HF reads to the total picoeukaryotic signal varied between water column zones (Fig. 1A). Most surface and DCM samples showed similar contributions (median values of $11 \%$ and 5\%, respectively), while a much larger dispersion was found in deeper samples, with median values of $3 \%$ in the mesopelagic and as large as $28 \%$ in the bathypelagic layer. In all depth zones, Alveolates (mainly Marine Alveolates [MALV] clades) and other Rhizarians (mainly radiolarians) represented $58-89 \%$ of the protist signal (Supporting Information Fig. S3). Regarding Shannon estimates of alpha diversity based on HF ASVs, there was a clear decreasing trend with depth, with very different Shannon values at surface and the bathypelagic (median of 4.3 and 1.1, respectively) (Fig. 1B). The taxonomic affiliation of ASVs allowed delineation of the contribution of different taxonomic groups to HF assemblages. Surface and DCM samples were composed of several groups with similar overall relative abundances (MAST-3, MAST-1, MAST-4, Picozoa, Chrysophyceae, and Bicosoecida; $9-16 \%$ on average each; Fig. 1C). At the sample level, these were relatively stable across the ocean, with Chrysophyceae and Bicosoecida deviating from the general trend (Supporting Information Fig. S4). In mesopelagic and bathypelagic zones, samples were overwhelmingly dominated by Chrysophyceae and Bicosoecida (25-68\% on average each; Fig. 1C) and showed distinct profiles in each station (Supporting Information Fig. S5).

We next compared the HF taxonomic composition derived from DNA samples (Fig. 1C) with that obtained from other
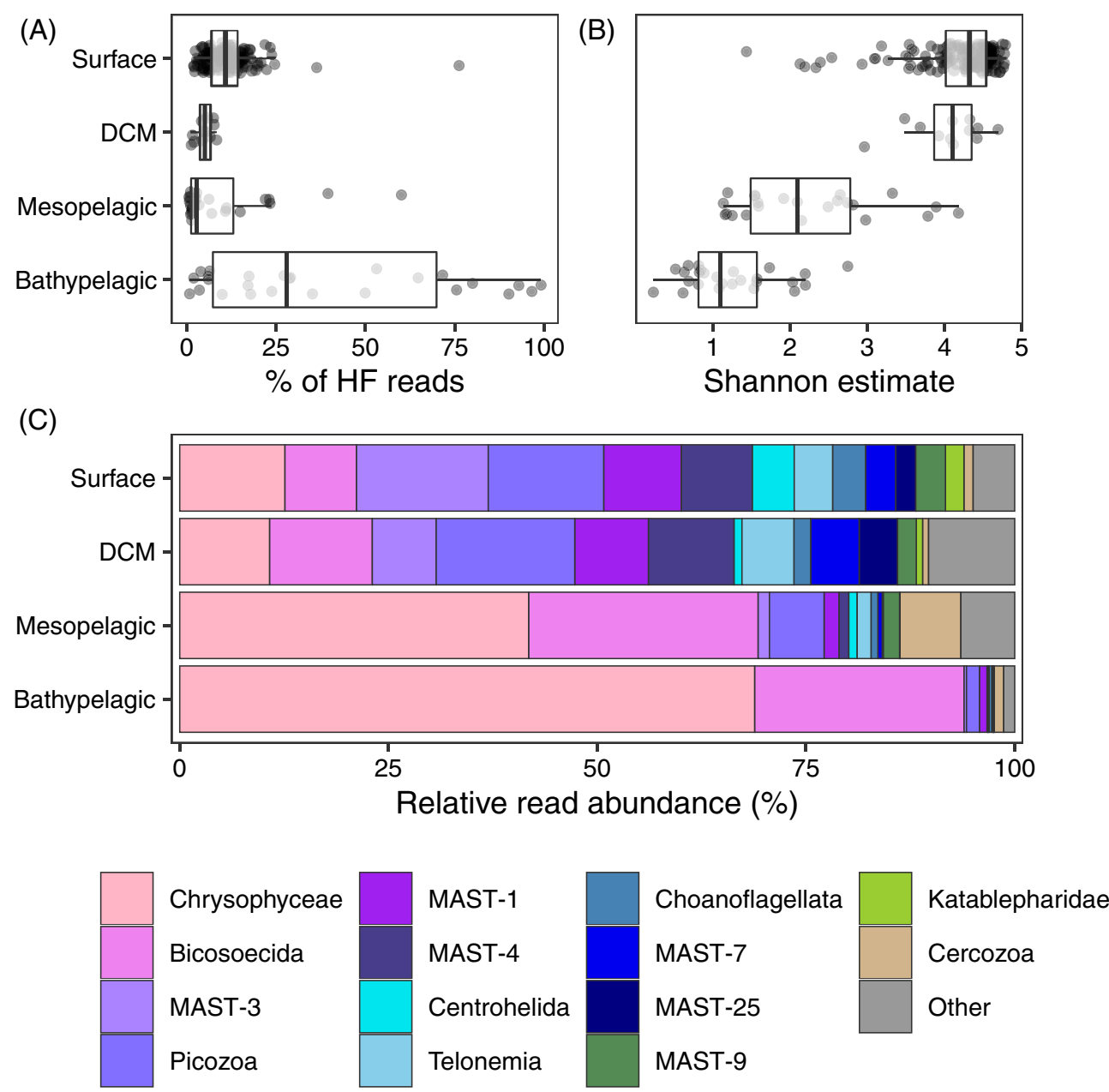

Fig. 1. Contribution of HFs in four depth zones of the ocean water column derived from 188 Malaspina samples (V4 DNA data set). (A) Percentage of reads affiliating to HF taxa with respect to the total picoeukaryotic signal. (B) Shannon alpha-diversity indices of HF assemblages using the corresponding ASVs. (C) Relative read abundance of the main taxonomic groups of HFs. 
oceanic data sets (Supporting Information Table S1). The RNA samples from the Malaspina survey yielded a highly similar HF composition in the four surveyed zones of the water column (Fig. 2), and ASVs that were detected in both DNA and RNA extracts represented $94-98 \%$ of the reads (Supporting Information Fig. S6). In general, ASVs unique to the RNA dataset did not add new diversity, with notable exceptions within Choanoflagellata, InSedMAST (putative novel MAST clades), and MAST-3 (Supporting Information Table S3). We also processed two additional Malaspina data sets derived from DNA extracts: V9-18S amplicons and metagenomes (mTags). In surface samples, the relative read abundances of taxonomic groups were generally similar to the V4 DNA data set, except for a lower presence of Bicosoecida and the detection of Euglenozoa (Diplonemea and Kinetoplastida) at moderate abundances (Fig. 2). In aphotic samples, the presence of Euglenozoa increased substantially, to the point that Diplonemea was the most abundant group in mesopelagic metagenomes (34\% on average). Finally, we analyzed the HF composition derived from V9-18S amplicons in photic waters of the TARA oceans survey (Fig. 2). Despite different sampling locations and size fractions analyzed (0.2-3 $\mu \mathrm{m}$ in Malaspina, and $0.8-5 \mu \mathrm{m}$ in TARA), groups like MAST-1, $-3,-4$, and Picozoa showed similar relative abundances in both datasets. The most noticeable difference was the higher presence of Diplonemea in the TARA oceans survey (12\% at surface and 32\% in DCM). This was higher than that reported in the V9 and mTags Malaspina data sets and could be explained by the size fraction analyzed, as diplonemid cell sizes tend to exceed $3 \mu \mathrm{m}$.

\section{Environmental drivers to HF community structure}

We performed PERMANOVA analyses on the HF assemblages defined by ASVs to identify the environmental factors explaining the variance in their community structure (Supporting Information Table S4). In surface samples, temperature (16\%) and the sampled ocean (13\%) were the main factors driving HF community variation, while in vertical profiles, the depth zone accounted for $22 \%$ of variance, and the sampled ocean for 19\%. Within aphotic samples (mesopelagic and bathypelagic), the majority of the variance was explained by water masses (34\%).

To further investigate how water temperature influenced HF assemblages in surface samples, we built a PCoA using Bray-Curtis dissimilarities among HF assemblages (Fig. 3A). Surface samples were distributed in the PCoA plot along the first axis (21\% of variance explained) following a
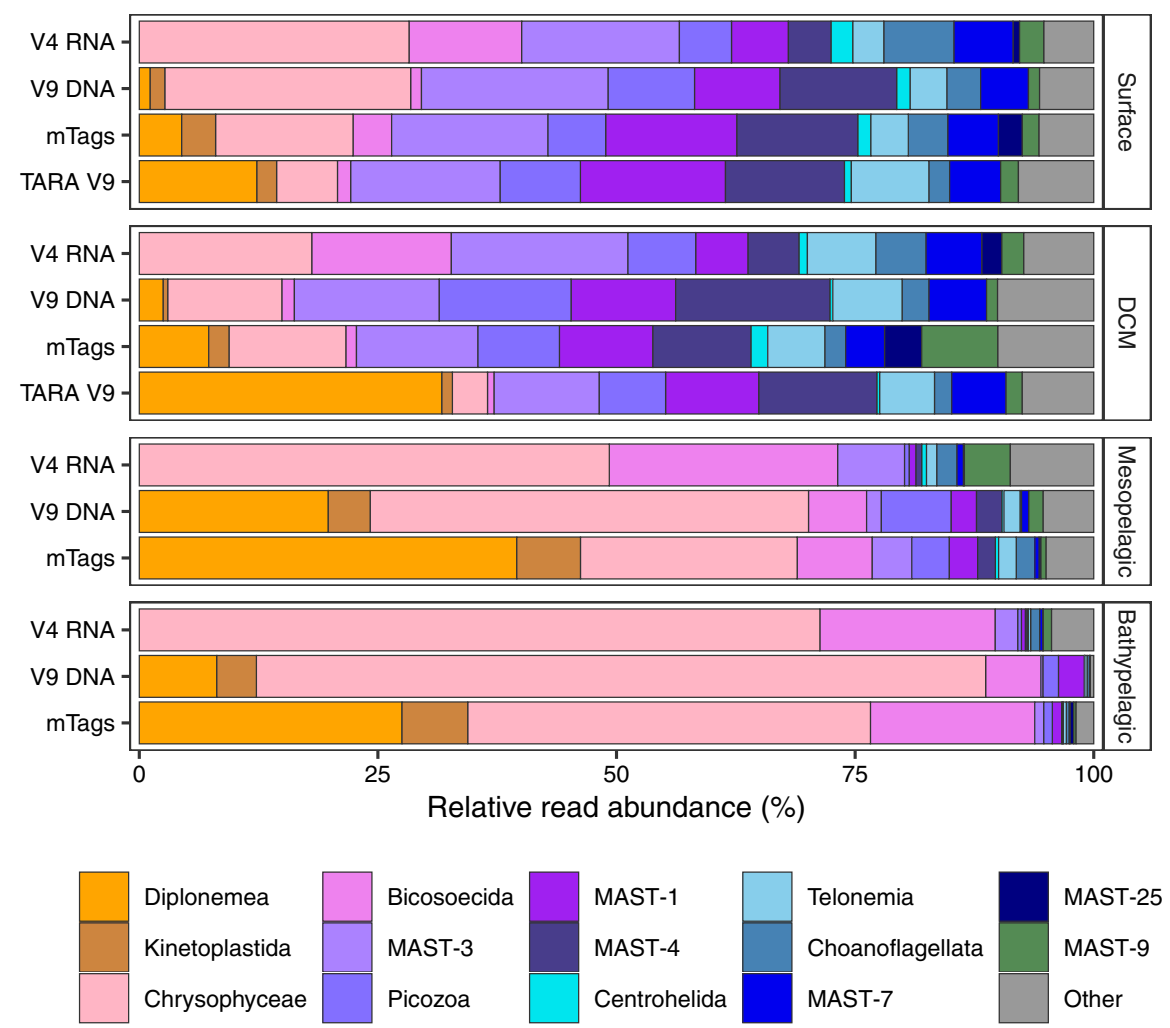

Fig. 2. Relative read abundance of the main taxonomic groups of HFs in four depth zones of the ocean as seen by different oceanic data sets. V4 RNA: Malaspina 18S-V4 amplicons from RNA extracts; V9 DNA: Malaspina 18S-V9 amplicons from DNA extracts; mTags: Malaspina 18S-V4 metagenomic tags; TARA V9: TARA oceans 18S-V9 amplicons from DNA extracts. See Supporting Information Table S1 for details of the number of samples and the fraction analyzed in each data set. Dictyochophyceae and Cercozoa (that had pigmented taxa) and InSedMAST (only defined in the V4 tree) were not included in this comparison. 
temperature gradient, thus highlighting the influence of this variable on the HF community structure. The effect of the sampled ocean was also evident when plotting the same dissimilarity matrix against the matrix of geographic distances among samples (Fig. 3B). Thus, geographically (and temporally) closer samples $(<1000 \mathrm{~km})$ were more similar, whereas dissimilarities increased up to distances of about $2000 \mathrm{~km}$ and remained fairly constant with samples that were farther apart.

\section{The dominant HFs in the ocean}

We identified which HF ASVs dominated in the different depth zones by averaging their relative read abundances in all samples from that zone. The above-mentioned pattern of decreasing diversity with increasing depth was clearly reflected by the number of ASVs that explained the majority of reads in each depth zone (Supporting Information Table S5). Thus, while 52 ASVs accounted for $60 \%$ of the signal at the surface, only 6 did so in the mesopelagic, and 3 in the bathypelagic. In surface samples, the ASVs contributing to HF assemblages appeared with a reasonable evenness (Table 1), and the 10 most abundant surface ASVs accounted for 2.0-3.5\% of the signal each: 1 ASV affiliated to Centrohelida (very distant to any cultured representative); 5 to MASTs (clades $-1,-3$ and -4 ); 1 to Chrysophyceae (Spumella sp. IOW86); 2 to Bicosoecida (Cafeteria burkhardae and Caecitellus pseudoparvulus); and 1 to Picozoa.

Some of these ASVs dominated at the DCM as well, where the most abundant ASVs were related to Picomonas judraskeda (7.4\%) and Caecitellus paraparvulus (6.2\%; Table 1). In deep waters, just two ASVs, namely Spumella IOW86 and C. burkhardae, dominated the HF assemblages and together accounted for $39.5 \%$ of the signal in mesopelagic and $53.9 \%$ in bathypelagic zones (Table 1). Looking at the list of dominant ASVs, it was evident that those related to cultured representatives increased with depth. A detailed list of the dominant ASVs in the four depth zones can be found in Supporting Information Table S6.

We then focused on the biogeography and distribution patterns of the 52 ASVs that explained $60 \%$ of the reads at the surface ocean (Fig. 4). These ASVs were found at comparable abundances in the RNA data set (Supporting Information Fig. S7) and also detected in the TARA survey (Supporting Information Table S7). Eleven ASVs were close (>97\% identity) to cultured species within Chrysophyceae, Bicosoecida, and Picozoa (albeit the reference culture is no longer available), 22 belonged to different environmental MAST clades, and the rest formed new taxa within groups known to contain cultured representatives (Fig. 4A). The majority of these dominant ASVs (31 out of 52) had a prevalence $>75 \%$. We classified each ASV into one of four distribution categories by analyzing (1) the ratio $\mathrm{SD} / \mathrm{mean}$ of their relative read abundances in all samples, (2) the differential abundance test across environmental factors, and (3) the heatmap clustering ASVs based on their normalized reads distribution (Supporting Information Fig. S8). ASVs were labeled as "equal" when they displayed relatively constant abundances across samples; "warm" or "cold" when showing preference for warmer or colder waters; and "patchy" when they appeared with peaks of high abundance in a few samples. More than half of the dominant surface ASVs (28) were labeled "equal," 16 were associated with temperature (7 "warm" and 9 "cold"), and 8 were "patchy" (Fig. 4A). All Bicosoecida ASVs fell into this latter category, as well as Spumella sp. and MAST-4E-sp1.

Unsurprisingly, patchy ASVs tended to have the lowest prevalence (minimum of $47 \%$ ). Analyzing the dominant ASVs in a phylogenetic tree (Fig. 4A), we detected closely related ones showing contrasted temperature distributions: MAST-4Bsp1 ("warm") and MAST-4A-sp1 ("cold") had 2 bp difference; and MAST-3A-sp1 ("warm") and MAST-3A-sp2 ("equal") had a single mismatch. We used the MicroMap web application developed here to construct global maps of the distribution of
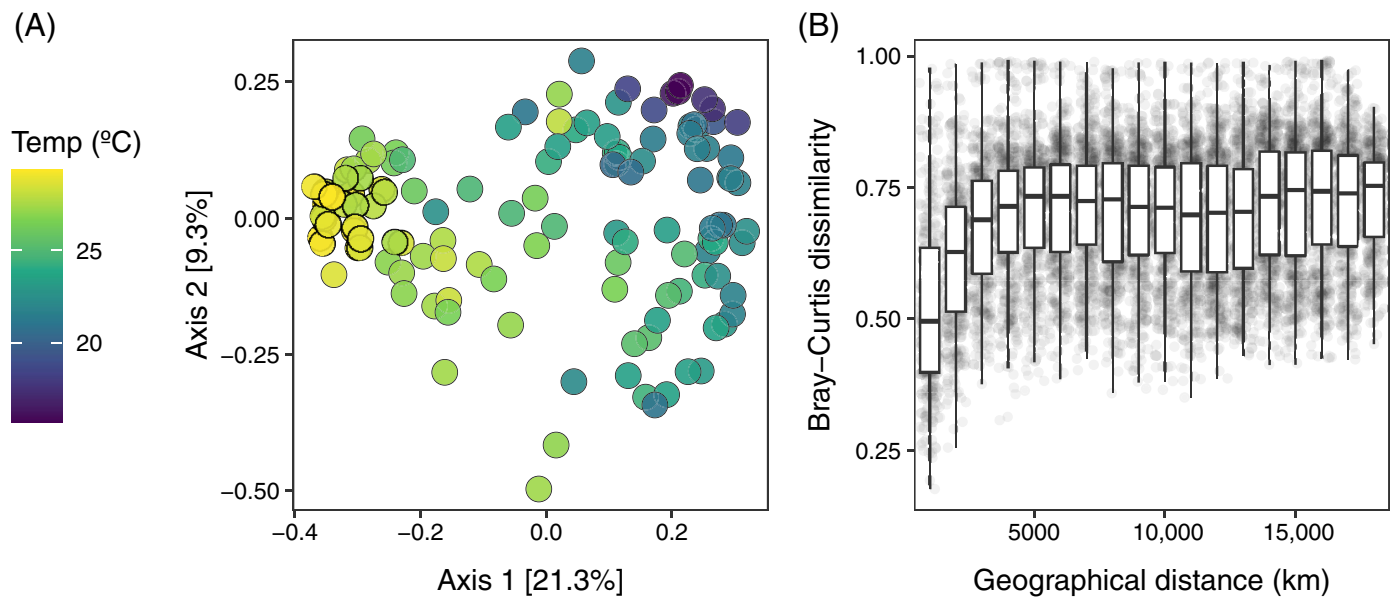

Fig. 3. Variation of HF community structure in surface samples. (A) PCoA of surface samples colored by water temperature based on Bray-Curtis dissimilarities among samples. (B) Plot of Bray-Curtis dissimilarity among samples against geographical distances. 
Table 1. Dominant HFs in the four depth zones of the ocean. The 10 ASVs with highest relative read abundance in each depth zone are displayed, including their mean relative read abundance (\%), SD, and prevalence (percentage of samples where it has been detected). Numbers at the beginning of ASV names correspond to their unique identifiers in the data set.

\begin{tabular}{|c|c|c|c|c|c|c|c|}
\hline \multicolumn{4}{|l|}{ Surface } & \multicolumn{4}{|l|}{ DCM } \\
\hline ASV & Mean(\%) & SD & $\operatorname{Prev}(\%)$ & ASV & Mean(\%) & SD & $\operatorname{Prev}(\%)$ \\
\hline 54/Centrohelida-sp1 & 3.5 & 3.8 & 97.6 & 138/Picomonas judraskeda (97.4\%) & 7.4 & 5.3 & 100.0 \\
\hline 75/MAST-1D-sp1 & 3.3 & 2.2 & 98.4 & 9/Caecitellus paraparvulus (97.7\%) & 6.2 & 14.3 & 83.3 \\
\hline 1/Spumella IOW86 (100\%) & 3.3 & 9.8 & 78.0 & 1/Spumella IOW86 (100\%) & 4.6 & 6.6 & 91.7 \\
\hline 4/Cafeteria burkhardae (100\%) & 3.2 & 7.6 & 80.3 & 31/MAST-4A-sp1 & 4.0 & 2.5 & 100.0 \\
\hline 31/MAST-4A-sp1 & 3.1 & 3.0 & 93.7 & 4/Cafeteria burkhardae (100\%) & 3.1 & 8.0 & 75.0 \\
\hline 51/Picozoa-sp1 & 2.7 & 2.5 & 98.4 & 75/MAST-1D-sp1 & 2.8 & 1.5 & 100.0 \\
\hline 76/MAST-1C-sp1 & 2.3 & 1.6 & 99.2 & 312/MAST-25-sp1 & 2.7 & 1.5 & 100.0 \\
\hline 36/Caecitellus pseudoparvulus (100\%) & 2.2 & 7.4 & 54.3 & 383/Picozoa-sp2 & 2.4 & 1.6 & 100.0 \\
\hline 110/MAST-4C-sp1 & 2.0 & 2.5 & 74.0 & 12/Caecitellus paraparvulus (100\%) & 2.2 & 4.9 & 50.0 \\
\hline 182/MAST-3A-sp2 & 2.0 & 2.4 & 97.6 & 763/MOCH-4-sp1 & 1.9 & 1.8 & 91.7 \\
\hline \multicolumn{4}{|l|}{ Mesopelagic } & \multicolumn{4}{|c|}{ Bathypelagic } \\
\hline ASV & Mean(\%) & SD & $\operatorname{Prev}(\%)$ & ASV & Mean(\%) & SD & $\operatorname{Prev}(\%)$ \\
\hline 1/Spumella IOW86 (100\%) & 29.3 & 31.7 & 100.0 & 1/Spumella IOW86 (100\%) & 38.7 & 36.8 & 100.0 \\
\hline 4/Cafeteria burkhardae (100\%) & 10.1 & 18.5 & 91.3 & 11/Spumella IOW86 (99.5\%) & 17.6 & 32.7 & 76.9 \\
\hline 12/Caecitellus paraparvulus (100\%) & 8.8 & 15.5 & 87.0 & 4/Cafeteria burkhardae (100\%) & 15.2 & 23.1 & 96.2 \\
\hline 9/Caecitellus paraparvulus (97.7\%) & 7.1 & 16.8 & 91.3 & 16/Chrysophyceae-sp1 & 6.7 & 10.6 & 80.8 \\
\hline 11/Spumella IOW86 (99.5\%) & 4.3 & 12.7 & 60.9 & 12/Caecitellus paraparvulus (100\%) & 4.2 & 9.5 & 84.6 \\
\hline 138/Picomonas judraskeda (97.4\%) & 3.9 & 6.1 & 95.7 & 9/Caecitellus paraparvulus (97.7\%) & 3.7 & 10.6 & 76.9 \\
\hline 16/Chrysophyceae-sp1 & 3.8 & 7.8 & 82.6 & 141/Paraphysomonas bandaiensis (100\%) & 1.8 & 8.3 & 11.5 \\
\hline 130/Helkesimastix sp. (99.7\%) & 2.0 & 9.4 & 17.4 & 36/Caecitellus pseudoparvulus (100\%) & 1.4 & 6.3 & 26.9 \\
\hline 123/Helkesimastix sp. (99.7\%) & 1.5 & 6.8 & 17.4 & 29/Planomonas micra (100\%) & 1.0 & 2.1 & 65.4 \\
\hline 1120/Cercozoa-sp1 & 1.1 & 1.5 & 91.3 & 686/Spumella IOW86 (99.7\%) & 0.8 & 0.8 & 92.3 \\
\hline
\end{tabular}

the dominant ASVs (Fig. 4B shows an example of each distribution pattern in surface samples). This provided a visual confirmation of the reported distribution characteristics: "cold" or "warm" ASVs occupied different regions of the cruise track (Supporting Information Fig. S1), "equal" ASVs showed a widespread distribution, and "patchy" ASVs displayed isolated peaks of high abundance.

\section{Co-occurrence of HF and prokaryotes at the surface ocean}

We performed a co-occurrence analysis between ASVs of HFs and prokaryotes retrieved from 113 surface samples of the Malaspina data set. After removing weak correlations $(<0.3)$ and putative indirect associations driven by environmental factors, the obtained network contained $479 \mathrm{HF}$ and 462 prokaryotic nodes (ASVs) and a total of 26,835 edges (correlations). The topology of the network displayed two clearly differentiated clusters driven by temperature, as clearly seen when coloring the nodes by thermal preference (Fig. 5A), leaving a still substantial number of nonresponsive ASVs. This two-cluster scheme was maintained in the subnetwork formed by the dominant $52 \mathrm{HF}$ ASVs and their correlations with prokaryotes (Fig. 5B). Most HF nodes were connected to several prokaryotic nodes, and these connections did not follow a specific taxonomic pattern. We then constructed a heatmap to visualize the specific co-occurrences, which clearly showed two clusters of HF nodes with opposite thermal preferences. These, in turn, were each associated with a cluster of prokaryotic nodes sharing the same thermal preference (Fig. 5C).

When looking at the composition of the correlating prokaryotes, the two thermal clusters included ASVs from similar groups: SAR11, SAR86, SAR116, Actinomarinales, and Prochlorococcus. For the latter two groups, the ASVs shown in the networks accounted for most of their overall environmental signal. ASV_41 and ASV_4, both related to Prochlorococcus, showed the highest correlations with HF ASVs in the cluster with preference for higher temperatures, while in the cluster with preference for colder waters ASV_209, from Candidatus Actinomarina, and ASV_382, from the SAR86 clade, were the taxa with the strongest correlations. Apart from these two main thermal clusters, some other correlations appeared. Thus, C. pseudoparvulus, which had a patchy distribution in the ocean, was nonetheless strongly correlated to ASV_2 (Rhodococcus), ASV_55 (Rubrivirga), and ASV_13 (WPS-2). These 
(A)

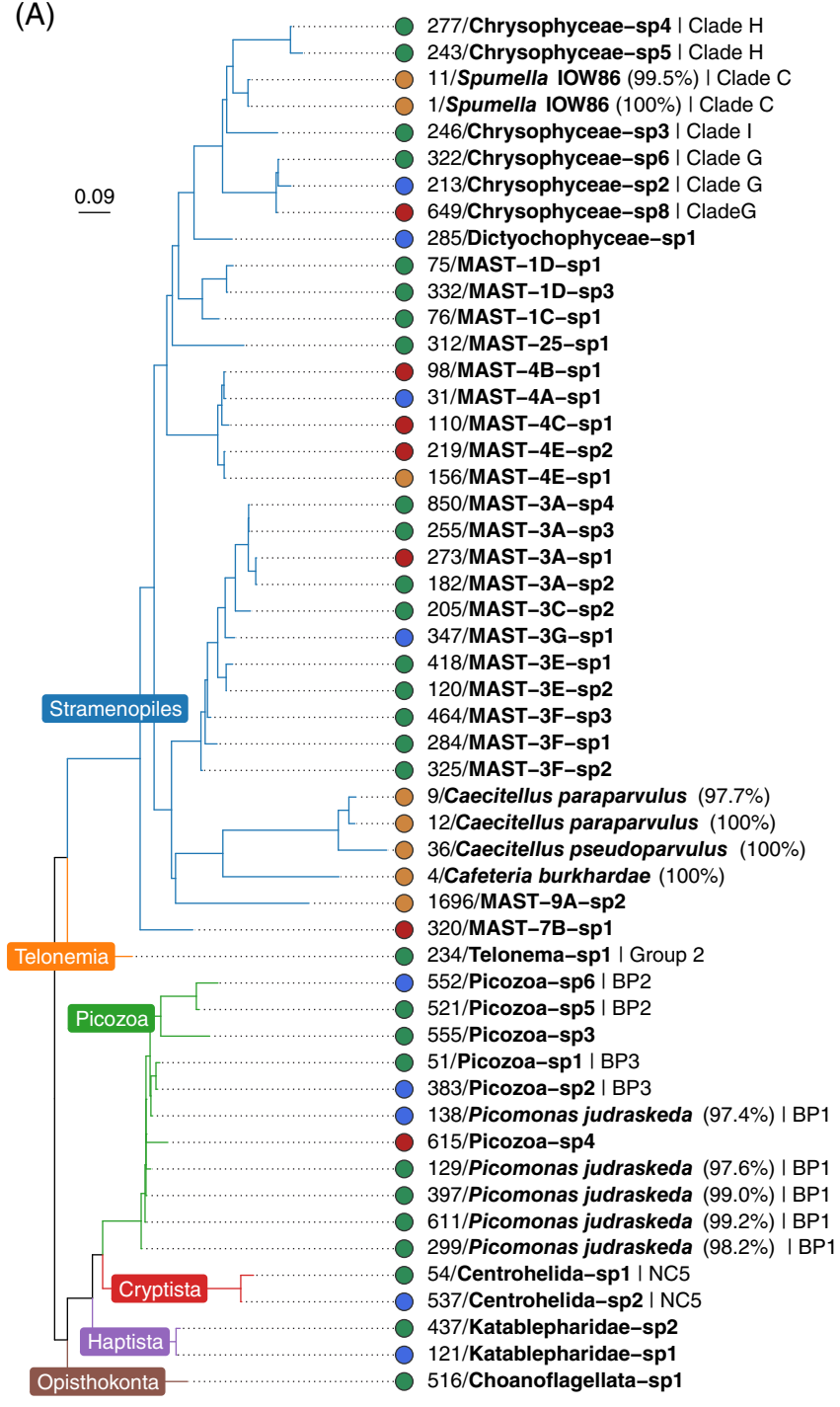

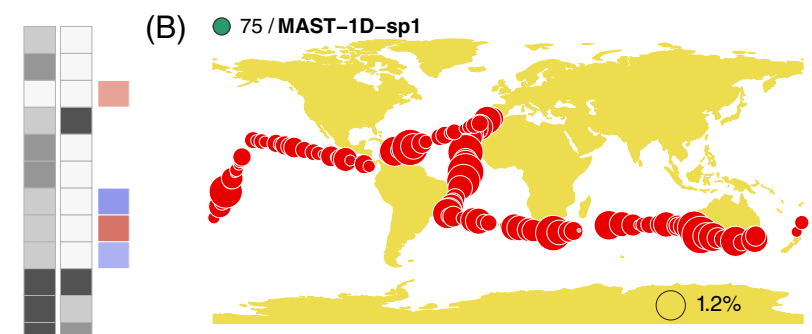
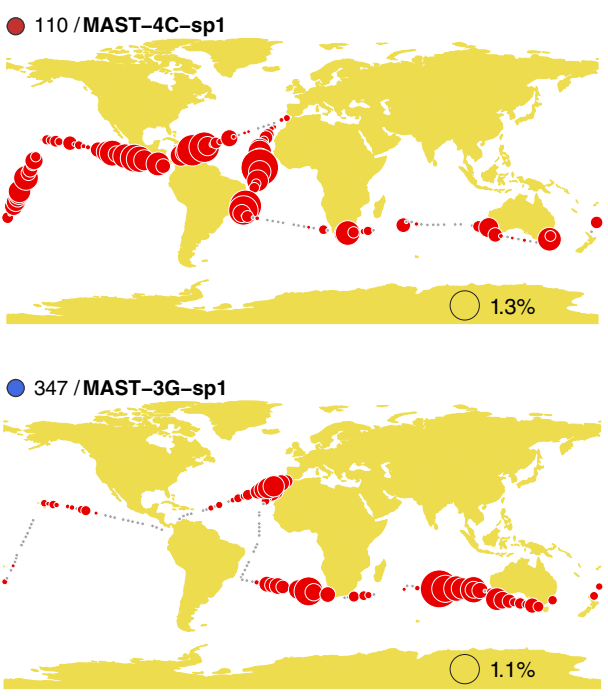

4 / Cafeteria burkhardae (100\%)

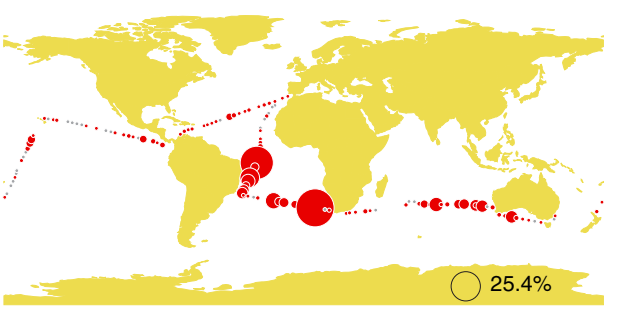

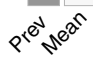

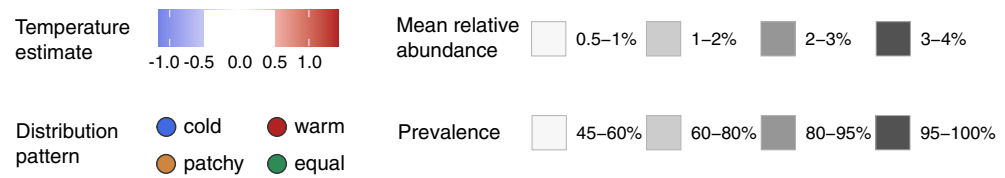

Fig. 4. The 52 dominant HF ASVs in surface waters of the ocean. (A) Phylogenetic tree and broad distributional features of each dominant ASV. Prevalence and mean relative abundance are displayed, as well as their estimates of a differential abundance test with temperature. Taxa are then colored according to their distribution pattern. (B) Biogeography maps for four dominant HF ASVs exhibiting differentiated distribution patterns obtained by the MicroMap web application (https://micromap.icm.csic.es/). The area of red circles is proportional to the relative read abundance of each ASV in the whole picoeukaryotic pool (maximum value is displayed in each panel), while gray circles indicate absence. Numbers at the beginning of ASV names correspond to their unique identifiers in the data set.

associations appeared isolated from the rest of the nodes in the co-occurrence network (Fig. 5B).

\section{Discussion}

One of the main issues that prevented us from obtaining a detailed assessment on the ecology of HFs, a key microbial component in marine ecosystems, is the lack of cultured strains that effectively represent the dominant HF species in the ocean. Besides the prevalent culturing bias, this was due to our general ignorance of which HF taxa dominate in the marine plankton, and whether or not this dominance is similar over oceanic biomes. In this study, we investigated the 

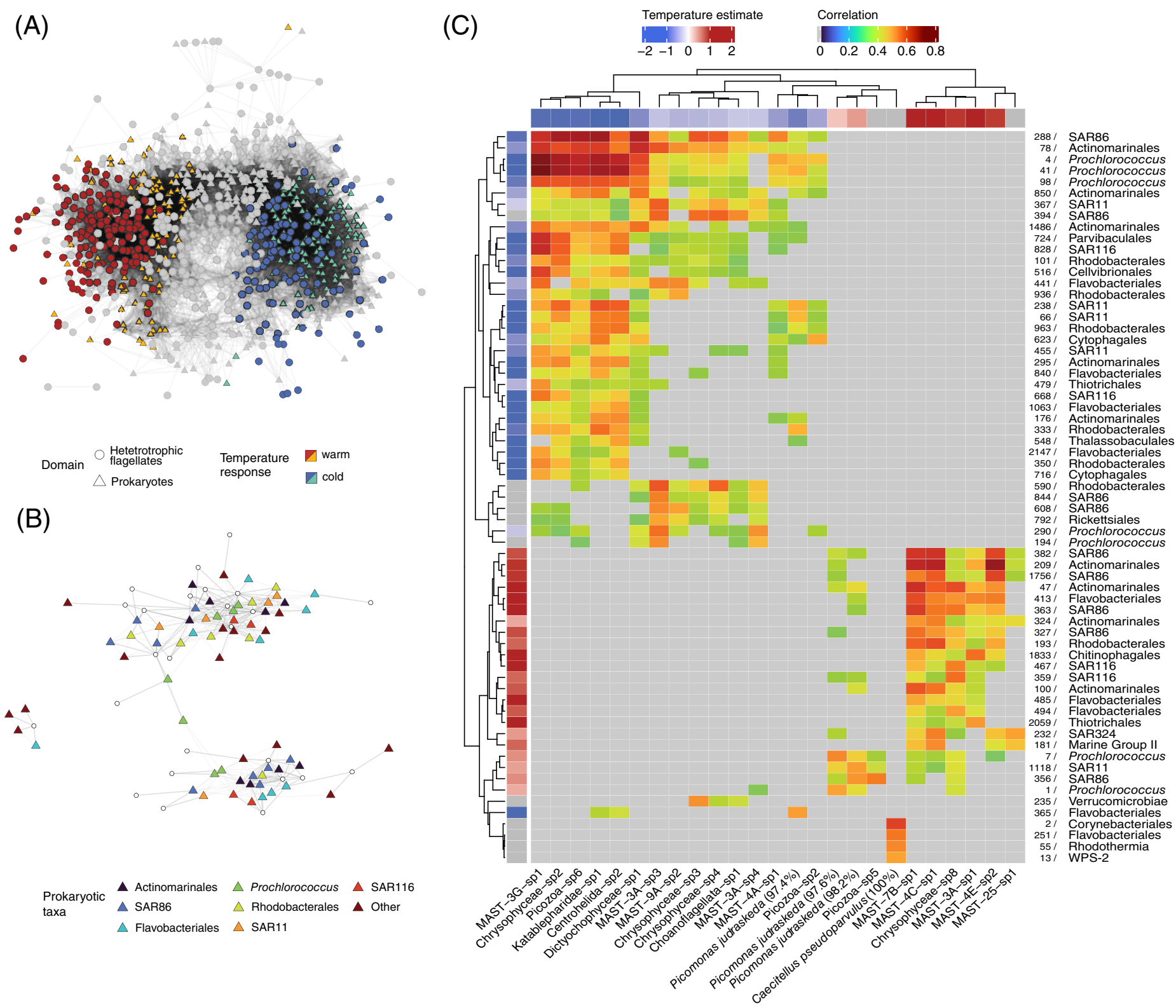

Fig. 5. Co-occurrence patterns between HF ASVs and prokaryotic ASVs at the surface of the ocean. (A) Co-occurrence network of HF and prokaryotic taxa. Each point represents an ASV, and these are colored by their temperature preference according to a differential abundance test (gray is a nonsignificant response to temperature). (B) Co-occurrence subnetwork of the dominant HF ASVs and prokaryotes, the latter colored by taxonomy. From the 52 dominant HF, only 25 display significant correlations and are thus shown in the subnetwork. (C) Heatmap displaying the correlations between dominant HFs (columns) and prokaryotes (rows); the estimates of a differential abundance test with temperature are displayed for each ASV.

presence of marker genes of HF taxa over the tropical and subtropical ocean using mostly Malaspina data (Giner et al. 2020; Logares et al. 2020; Obiol et al. 2020) together with Tara Oceans data (de Vargas et al. 2015). While abundance of ribosomal marker genes may not always correlate directly with abundance of cells due to copy number variations ( $\mathrm{Zhu}$ et al. 2005), these could be a good proxy for the in situ abundance of the HFs analyzed here, as small-sized cells are hypothesized to contain a low and constrained number of
rDNA copies (Zhu et al. 2005; Rodríguez-Martínez et al. 2009). With this, we show the prevalence of particular taxonomic groups over horizontal and vertical scales, and identify a subset of dominant and widespread HF taxa in different depth zones of the water column of the ocean.

In surface waters, the dominant HFs resolved into two clearly differentiated groups with distinct distributions. The first group formed a constant "core" community of uncultured taxa belonging to MAST clades (mostly $-1,-3$, and -4 ), Picozoa, and 
Chrysophyceae (uncultured clades G, H, and I) that were found in most samples, some of them with a preference for colder or warmer waters. Previous microscopic counts confirmed the relatively homogeneous cell abundance in Malaspina surface samples of relevant clades like MAST-4, MAST-1C, and MAST-7, which averaged between 10 and 50 cells $\mathrm{mL}^{-1}$ (Mangot et al. 2018). Moreover, these core surface taxa were scarce in the deep and dark ocean. A recent study using single amplified genomes detected rhodopsin genes in most MAST species investigated (Labarre et al. 2021), which could partially explain their success in photic waters and their virtual absence in the dark ocean. Although no cultured representative exists for the dominant MASTs, there are already partial genomes for 10 of them (Labarre et al. 2021), and this will be an invaluable resource for further research. The high number of picozoans among the dominant surface ASVs suggests that these likely play a significant role in the ecosystem, a role that is yet to be characterized (Burki et al. 2020). The only described picozoan species, $P$. judraskeda, originally isolated from coastal waters (Seenivasan et al. 2013) but today lost, was not detected in the open ocean. Finally, the most widespread and abundant ASV in surface waters was Centrohelida-sp1. This ASV, together with many others, formed an environmental clade phylogenetically distant to the described species of centrohelids (Shishkin et al. 2018). The prevalence of Centrohelida-sp1 in the picoeukaryotic fraction in surface waters hints at the existence of undescribed centrohelids that might be smaller than those reported (i.e., Oxnerella micra; Cavalier-Smith and Chao 2012) and could be important bacterivores.

Parallel to the described "core" community, surface waters contained a second set of patchy, unevenly distributed HF taxa from the groups Chrysophyceae (genus Spumella in clade C), and Bicosoecida (species C. burkhardae, and C. paraparvulus). These ASVs were markedly dominant in deeper aphotic zones and coincided with a clear decrease in HF alpha diversity, a pattern already seen in studies targeting all microbial eukaryotes (Schnetzer et al. 2011; Giner et al. 2020). The low diversity and high dominance of these species, known to have a high tolerance to pressure (Živaljić et al. 2018) and to be well adapted to a particle-associated lifestyle (Jeuck and Arndt 2013), may be explained by the fact that microbial life in the nutrient-poor deep ocean greatly relies on the particulate organic matter flux from upper layers (Nagata et al. 2010). So, the patchy distribution at the surface and deep ocean of Spumella, Cafeteria, and Caecitellus spp. may be due to their living in or on particles as nutrient-rich hotspots. The fact that we detected them in the $0.2-3 \mu \mathrm{m}$ size fraction could be due to the fragility of marine particles, which could break during filtration (Bochdansky et al. 2017). The particle-attached existence of these taxa could also explain their relative ease of culturing compared to free-living species, since standard culturing media (i.e., a rice grain with seawater) could somehow resemble nutrient-rich particles (del Campo et al. 2013). In fact, while in the deep ocean the majority of dominant ASVs here detected seem to be already in culture, no cultured representative exists for the dominant surface ASVs besides these few patchy species, highlighting the current bias that exists in protistan knowledge (Keeling and Burki 2019).

Together with Chrysophyceae and Bicosoecida, Diplonemea (and to a lower extent Kinetoplastida) also appeared to be major contributors to deep HF assemblages. This was obvious when analyzing V9 amplicons and metagenomes, but unfortunately these euglenozoans did not appear in the V4-18S data set, as the combination of longer V4 regions (Pernice et al. 2016) and several mismatches with universal primers (Vaulot et al. 2021) prevent their correct amplification. Therefore, these euglenozoans do not appear in the catalogue of ASVs, a possibly minor issue for surface assemblages but an important limitation for the deep survey. The importance of deep diplonemids agrees with previous reports (Lara et al. 2009; Flegontova et al. 2016; Schoenle et al. 2021), albeit it has been also proposed that read abundances could overestimate cell abundances, given the tendency of Diplonemea to display high rDNA copy numbers (Mukherjee et al. 2020).

Environmental parameters measured during the Malaspina cruise could explain part of the variation displayed by $\mathrm{HF}$ assemblages along horizontal and vertical scales. Increasing depth in the water column resulted in a clear drop of HF diversity and the disappearance of many surface taxonomic groups, with deep ocean assemblages being dominated by only a few of them. In vertical profiles, the main explanatory variables were depth in the water column and the sampled ocean, together with water masses when aphotic samples were processed separately, as reported before for whole picoeukaryotic communities (Giner et al. 2020). In surface waters, we also detected the effect of the sampled ocean, but temperature was the primary environmental factor shaping sunlit HF communities. In global studies with strong latitudinal gradients, temperature has a marked effect in most planktonic groups (Ibarbalz et al. 2019), and HF assemblages are not an exception (Patterson and Lee 2000; Azovsky et al. 2016). The temperature effect was also detected in studies targeting specific HF taxa (Boenigk et al. 2006; RodríguezMartínez et al. 2013; Flegontova et al. 2020; Latorre et al. 2021). Despite samples analyzed here having a relatively narrow temperature range $\left(16-29^{\circ} \mathrm{C}\right)$, we could detect a temperature effect on HF assemblages, highlighting the possible alterations that these could face by the expected increase of sea surface temperature due to global warming (Hutchins and Fu 2017). The temperature gradient reported in the Malaspina survey was dominated by latitudinal changes and not by putative seasonality of the sampled sites, as could be inferred in Supporting Information Fig. S1 (i.e., samples from the same latitude that were collected several months apart share highly similar temperature values). Nonetheless, in our work, environmental variables were incomplete predictors of community structure (at surface, they only predicted $36 \%$ of the variance). Biotic factors, such as the community composition of prokaryotic assemblages, could be playing an important role in shaping HF assemblages. 
To reveal putative biotic interactions, we assessed the cooccurrence between $\mathrm{HF}$ and prokaryotic taxa in association networks. We did not aim to use co-occurrence as a direct proxy of interaction (Blanchet et al. 2020), but to identify potentially interesting biotic relationships that would then need to be experimentally validated (Carr et al. 2019). Even after removing associations that could arise from shared environmental preferences (Röttjers and Faust 2018; Deutschmann et al. 2021), HF and prokaryotic ASVs were placed in two differentiated clusters formed by abundant taxa with different thermal preferences, a phenomenon already seen in previous studies (Pommier et al. 2007; Fuhrman et al. 2008; LimaMendez et al. 2015). At a broad scale, specific HF did not show preferential correlations with specific prokaryotic taxa, and this agrees with the perception that cell size is the main factor in prey vulnerability (Jürgens and Massana 2008). However, some of the correlations displayed by taxa not belonging to the main "warm" and "cold" clusters could also suggest prey preference by some HFs. In fact, it has been shown that feeding natural HF communities with different bacterial strains can select distinct assemblages in freshwater manipulations (Šimek et al. 2018), and that closely related grazers can differently impact bacterial communities (Glücksman et al. 2010). Our results thus represent an opportunity to further study particular predator-prey interactions in new experimental scenarios.

Having identified the putatively most relevant HFs in the ocean, a renewed effort to culture them should be made, as these could be developed into new model organisms to be used to gain a better understanding of the role of HFs in the ecosystem. Single cell genomics is also a promising alternative to capture the genomes of these dominant species (Labarre et al. 2021), which can then be used in comparative genomics or to interpret complex metagenomic and metatranscriptomic data sets. Overall, our work paves the road for future studies on marine HFs, highlighting key species that may be playing crucial roles in the plankton.

\section{Data availability statement}

Raw data used in this study can be found at the European Nucleotide Archive (http://www.ebi.ac.uk/ena) with accession numbers PRJEB23913 and PRJEB25224 (surface data sets for 18S and 16S, respectively; Logares et al. 2020), and PRJEB23771 (vertical profiles; Giner et al. 2020). TARA Oceans (de Vargas et al. 2015; Callahan 2017) and Malaspina V9/mTags tables (Obiol et al. 2020) can be found at Zenodo with DOIs 10.5281/ zenodo.581694 and 10.5281/zenodo.3629394, respectively. All tables and code used for data processing and analyses can be found at https://github.com/aleixop/Malaspina_HF.

\section{References}

Adl, S. M., and others. 2019. Revisions to the classification, nomenclature, and diversity of eukaryotes. J. Eukaryot. Microbiol. 66: 4-119. doi:10.1111/jeu.12691
Altschul, S. F., W. Gish, W. Miller, E. W. Myers, and D. J. Lipman. 1990. Basic local alignment search tool. J. Mol. Biol. 215: 403-410. doi:10.1016/S0022-2836(05)80360-2

Azam, F., T. Fenchel, J. Field, J. Gray, L. Meyer-Reil, and F. Thingstad. 1983. The ecological role of water-column microbes in the sea. Mar. Ecol. Prog. Ser. 10: 257-263. doi: 10.3354/meps010257

Azovsky, A. I., D. V. Tikhonenkov, and Y. A. Mazei. 2016. An estimation of the global diversity and distribution of the smallest eukaryotes: Biogeography of marine benthic heterotrophic flagellates. Protist 167: 411-424. doi:10.1016/j. protis.2016.07.001

Bass, D., and others. 2018. Rhizarian 'Novel Clade 10' revealed as abundant and diverse planktonic and terrestrial flagellates, including Aquavolon n. gen. J. Eukaryot. Microbiol. 65: 828-842. doi:10.1111/jeu.12524

Blanchet, F. G., K. Cazelles, and D. Gravel. 2020. Co-occurrence is not evidence of ecological interactions. Ecol. Lett. 23: 1050-1063. doi:10.1111/ele.13525

Bochdansky, A. B., M. A. Clouse, and G. J. Herndl. 2017. Eukaryotic microbes, principally fungi and labyrinthulomycetes, dominate biomass on bathypelagic marine snow. ISME J. 11: 362-373. doi:10.1038/ismej.2016.113

Boenigk, J., K. Pfandl, T. Garstecki, H. Harms, G. Novarino, and A. Chatzinotas. 2006. Evidence for geographic isolation and signs of endemism within a protistan morphospecies. Appl. Environ. Microbiol. 72: 5159-5164. doi:10.1128/ AEM.00601-06

Burki, F., A. J. Roger, M. W. Brown, and A. G. B. Simpson. 2020. The new tree of eukaryotes. Trends Ecol. Evol. 35: 43-55. doi:10.1016/j.tree.2019.08.008

Callahan, B. J. 2017. ASV tables inferred by DADA2 from the TARA Oceans v9 metabarcoding dataset. doi:10.5281/ zenodo. 581694

Callahan, B. J., P. J. McMurdie, M. J. Rosen, A. W. Han, A. J. A. Johnson, and S. P. Holmes. 2016. DADA2: High-resolution sample inference from Illumina amplicon data. Nat. Methods 13: 581-583. doi:10.1038/nmeth.3869

Canals, O., A. Obiol, I. Muhovic, D. Vaqué, and R. Massana. 2020. Ciliate diversity and distribution across horizontal and vertical scales in the open ocean. Mol. Ecol. 29: 28242839. doi: $10.1111 /$ mec. 15528

Carr, A., C. Diener, N. S. Baliga, and S. M. Gibbons. 2019. Use and abuse of correlation analyses in microbial ecology. ISME J. 13: 2647-2655. doi:10.1038/s41396-019-0459-z

Cavalier-Smith, T., and E. E. Chao. 2012. Oxnerella micra sp. n. (Oxnerellidae fam. n.), a tiny naked centrohelid, and the diversity and evolution of heliozoa. Protist 163: 574601. doi:10.1016/j.protis.2011.12.005

de Vargas, C., and others. 2015. Eukaryotic plankton diversity in the sunlit ocean. Science 348: 1261605. doi:10.1126/ science.1261605

del Campo, J., and R. Massana. 2011. Emerging diversity within chrysophytes, choanoflagellates and bicosoecids 
based on molecular surveys. Protist 162: 435-448. doi:10. 1016/j.protis.2010.10.003

del Campo, J., V. Balagué, I. Forn, I. Lekunberri, and R. Massana. 2013. Culturing bias in marine heterotrophic flagellates analyzed through seawater enrichment incubations. Microb. Ecol. 66: 489-499. doi:10.1007/s00248-0130251-y

Deutschmann, I. M., G. Lima-Mendez, A. K. Krabberød, J. Raes, K. Faust \& R. Logares 2019. EnDED - environmentally-driven edge detection program. doi:10.5281/ zenodo. 3271730

Deutschmann, I. M., A. K. Krabberød, L. F. Benites, and others. 2021. Disentangling temporal associations in marine microbial networks. bioRxiv 2021.07.13.452187. doi:10. 1101/2021.07.13.452187

Diez, B., C. Pedrós-Alió, and R. Massana. 2001. Study of genetic diversity of eukaryotic picoplankton in different oceanic regions by small-subunit rRNA gene cloning and sequencing. Appl. Environ. Microbiol. 67: 2932-2941. doi: 10.1128/AEM.67.7.2932-2941.2001

Edgcomb, V. P., D. T. Kysela, A. Teske, A. de Vera Gomez, and M. L. Sogin. 2002. Benthic eukaryotic diversity in the Guaymas Basin hydrothermal vent environment. Proc. Natl. Acad. Sci. USA 99: 7658-7662. doi:10.1073/pnas. 062186399

Fenchel, T. 1986. The ecology of heterotrophic microflagellates, p. 57-97. In K. C. Marshall [ed.], Advances in microbial ecology. Springer.

Flegontova, O., and others. 2016. Extreme diversity of diplonemid eukaryotes in the ocean. Curr. Biol. 26: 30603065. doi:10.1016/j.cub.2016.09.031

Flegontova, O., P. Flegontov, S. Malviya, J. Poulain, C. de Vargas, C. Bowler, J. Lukeš, and A. Horák. 2018. Neobodonids are dominant kinetoplastids in the global ocean. Environ. Microbiol. 20: 878-889. doi:10.1111/14622920.14034

Flegontova, O., P. Flegontov, P. A. C. Londoño, W. Walczowski, D. Šantić, V. P. Edgcomb, J. Lukeš, and A. Horák. 2020. Environmental determinants of the distribution of planktonic diplonemids and kinetoplastids in the oceans. Environ. Microbiol. 22: 4014-4031. doi:10.1111/ 1462-2920.15190

Friedman, J., and E. J. Alm. 2012. Inferring correlation networks from genomic survey data. PLoS Comput. Biol. 8: e1002687. doi:10.1371/journal.pcbi.1002687

Fuhrman, J. A., and R. T. Noble. 1995. Viruses and protists cause similar bacterial mortality in coastal seawater. Limnol. Oceanogr. 40: 1236-1242. doi:10.4319/lo.1995.40. 7.1236

Fuhrman, J. A., J. A. Steele, I. Hewson, M. S. Schwalbach, M. V. Brown, J. L. Green, and J. H. Brown. 2008. A latitudinal diversity gradient in planktonic marine bacteria. Proc. Natl. Acad. Sci. USA 105: 7774-7778. doi:10.1073/pnas. 0803070105
Giner, C. R., M. C. Pernice, V. Balagué, C. M. Duarte, J. M. Gasol, R. Logares, and R. Massana. 2020. Marked changes in diversity and relative activity of picoeukaryotes with depth in the world ocean. ISME J. 14: 437-449. doi:10. 1038/s41396-019-0506-9

Gloor, G. B., J. M. Macklaim, V. Pawlowsky-Glahn, and J. J. Egozcue. 2017. Microbiome datasets are compositional: And this is not optional. Front. Microbiol. 8: 1-6. doi:10. 3389/fmicb.2017.02224

Glücksman, E., T. Bell, R. I. Griffiths, and D. Bass. 2010. Closely related protist strains have different grazing impacts on natural bacterial communities. Environ. Microbiol. 12: 3105-3113. doi:10.1111/j.1462-2920.2010.02283.x

Guillou, L., M. Viprey, A. Chambouvet, R. M. Welsh, A. R. Kirkham, R. Massana, D. J. Scanlan, and A. Z. Worden. 2008. Widespread occurrence and genetic diversity of marine parasitoids belonging to Syndiniales (Alveolata). Environ. Microbiol. 10: 3349-3365. doi:10.1111/j.14622920.2008.01731.x

Hutchins, D. A., and F. Fu. 2017. Microorganisms and ocean global change. Nat. Microbiol. 2: 17058. doi:10.1038/ nmicrobiol.2017.58

Ibarbalz, F. M., and others. 2019. Global trends in marine plankton diversity across kingdoms of life. Cell 179: 10841097. doi:10.1016/j.cell.2019.10.008

Jeuck, A., and H. Arndt. 2013. A short guide to common heterotrophic flagellates of freshwater habitats based on the morphology of living organisms. Protist 164: 842-860. doi: 10.1016/j.protis.2013.08.003

Jürgens, K., and R. Massana. 2008. Protistan grazing on marine bacterioplankton, p. 383-441. In D. L. Kirchman [ed.], Microbial ecology of the oceans. John Wiley \& Sons.

Katoh, K., and D. M. Standley. 2013. MAFFT multiple sequence alignment software version 7: Improvements in performance and usability. Mol. Biol. Evol. 30: 772-780. doi:10.1093/molbev/mst010

Keeling, P. J., and F. Burki. 2019. Progress towards the tree of eukaryotes. Curr. Biol. 29: R808-R817. doi:10.1016/j.cub. 2019.07.031

Kozlov, A. M., D. Darriba, T. Flouri, B. Morel, and A. Stamatakis. 2019. RAxML-NG: A fast, scalable and userfriendly tool for maximum likelihood phylogenetic inference. Bioinformatics 35: 4453-4455. doi:10.1093/ bioinformatics/btz305

Labarre, A., and others. 2021. Comparative genomics reveals new functional insights in uncultured MAST species. ISME J. 15: 1767-1781. doi:10.1038/s41396-02000885-8

Lara, E., D. Moreira, A. Vereshchaka, and P. López-García. 2009. Pan-oceanic distribution of new highly diverse clades of deep-sea diplonemids. Environ. Microbiol. 11: 47-55. doi:10.1111/j.1462-2920.2008.01737.x

Latorre, F., and others. 2021. Niche adaptation promoted the evolutionary diversification of tiny ocean predators. Proc. 
Natl. Acad. Sci. USA 118: e2020955118. doi:10.1073/pnas. 2020955118

Le Bescot, N., and others. 2016. Global patterns of pelagic dinoflagellate diversity across protist size classes unveiled by metabarcoding. Environ. Microbiol. 18: 609-626. doi: 10.1111/1462-2920.13039

Lima-Mendez, G., and others. 2015. Determinants of community structure in the global plankton interactome. Science 348: 1262073. doi:10.1126/science. 1262073

Logares, R., and others. 2020. Disentangling the mechanisms shaping the surface ocean microbiota. Microbiome 8: 1-17. doi:10.1186/s40168-020-00827-8

Lopes dos Santos, A., P. Gourvil, M. Tragin, M.-H. Noël, J. Decelle, S. Romac, and D. Vaulot. 2017. Diversity and oceanic distribution of prasinophytes clade VII, the dominant group of green algae in oceanic waters. ISME J. 11: 512 528. doi:10.1038/ismej.2016.120

López-García, P., F. Rodríguez-Valera, C. Pedrós-Alió, and D. Moreira. 2001. Unexpected diversity of small eukaryotes in deep-sea Antarctic plankton. Nature 409: 603-607. doi:10. 1038/35054537

Malviya, S., and others. 2016. Insights into global diatom distribution and diversity in the world's ocean. Proc. Natl. Acad. Sci. USA 113: E1516-E1525. doi:10.1073/pnas. 1509523113

Mangot, J., I. Forn, A. Obiol, and R. Massana. 2018. Constant abundances of ubiquitous uncultured protists in the open sea assessed by automated microscopy. Environ. Microbiol. 20: 3876-3889. doi:10.1111/1462-2920.14408

Martin, B. D., D. Witten, and A. D. Willis. 2020. Modeling microbial abundances and dysbiosis with beta-binomial regression. Ann. Appl. Stat. 14: 94-115. doi:10.1214/19AOAS1283

Martin, M. 2011. Cutadapt removes adapter sequences from high-throughput sequencing reads. EMBnet J 17: 10. doi: 10.14806/ej.17.1.200

Massana, R., J. Castresana, V. Balagué, L. Guillou, K. Romari, K. Valentin, and C. Pedrós-Alió. 2004. Phylogenetic and ecological analysis of novel marine stramenopiles. Appl. Environ. Microbiol. 70: 3528-3534. doi:10.1128/AEM.70.6. 3528

Massana, R., J. del Campo, M. E. Sieracki, S. Audic, and R. Logares. 2014. Exploring the uncultured microeukaryote majority in the oceans: Reevaluation of ribogroups within stramenopiles. ISME J. 8: 854-866. doi:10.1038/ismej. 2013.204

McMurdie, P. J., and S. Holmes. 2013. phyloseq: An R package for reproducible interactive analysis and graphics of microbiome census data. PLoS One 8: e61217. doi:10.1371/ journal.pone.0061217

Metz, S., D. Singer, I. Domaizon, F. Unrein, and E. Lara. 2019. Global distribution of Trebouxiophyceae diversity explored by high-throughput sequencing and phylogenetic approaches. Environ. Microbiol. 21: 3885-3895. doi:10. 1111/1462-2920.14738

Moon-van der Staay, S. Y., R. De Wachter, and D. Vaulot. 2001. Oceanic 18S rDNA sequences from picoplankton reveal unsuspected eukaryotic diversity. Nature 409: 607610. doi:10.1038/35054541

Moreira, D., and P. López-García. 2014. The rise and fall of picobiliphytes: How assumed autotrophs turned out to be heterotrophs. Bioessays 36: 468-474. doi:10.1002/bies.201300176

Mukherjee, I., and others. 2020. A freshwater radiation of diplonemids. Environ. Microbiol. 22: 4658-4668. doi:10. 1111/1462-2920.15209

Nagata, T., and others. 2010. Emerging concepts on microbial processes in the bathypelagic ocean - ecology, biogeochemistry, and genomics. Deep-Sea Res. II Top. Stud. Oceanogr. 57: 1519-1536. doi:10.1016/j.dsr2.2010.02.019

Not, F., K. Valentin, K. Romari, C. Lovejoy, R. Massana, K. Töbe, D. Vaulot, and L. K. Medlin. 2007. Picobiliphytes: A marine picoplanktonic algal group with unknown affinities to other eukaryotes. Science 315: 253-255. doi:10.1126/ science.1136264

Obiol, A., C. R. Giner, P. Sánchez, C. M. Duarte, S. G. Acinas, and R. Massana. 2020. A metagenomic assessment of microbial eukaryotic diversity in the global ocean. Mol. Ecol. Resour. 20: 718-731. doi:10.1111/1755-0998.13147

Oksanen, J., Blanchet, F. G., Friendly, M., and others. 2020. vegan: Community Ecology Package.

Patterson, D. J., and W. J. Lee. 2000. Geographic distribution and diversity of free-living heterotrophic flagellates, p. 267-287. In B. S. Leadbeater and J. C. Green [eds.], The flagellates: Unity, diversity and evolution. Taylor \& Francis.

Pedersen, T. L. 2020a. Tidygraph: A tidy API for graph manipulation.

Pedersen, T. L. 2020b. ggraph: An implementation of grammar of graphics for graphs and networks.

Pernice, M. C., C. R. Giner, R. Logares, J. Perera-Bel, S. G. Acinas, C. M. Duarte, J. M. Gasol, and R. Massana. 2016. Large variability of bathypelagic microbial eukaryotic communities across the world's oceans. ISME J. 10: 945-958. doi:10.1038/ismej.2015.170

Pernthaler, J. 2005. Predation on prokaryotes in the water column and its ecological implications. Nat. Rev. Microbiol. 3: 537-546. doi:10.1038/nrmicro1180

Pommier, T., B. Canbäck, L. Riemann, K. H. Boström, K. Simu, P. Lundberg, A. Tunlid, and A. Hagström. 2007. Global patterns of diversity and community structure in marine bacterioplankton. Mol. Ecol. 16: 867-880. doi:10.1111/j. 1365-294X.2006.03189.x

R Core Team 2020. R: A Language and Environment for Statistical Computing, R Foundation for Statistical Computing.

Rodríguez-Martínez, R., M. Labrenz, J. del Campo, I. Forn, K. Jürgens, and R. Massana. 2009. Distribution of the uncultured protist MAST-4 in the Indian Ocean, Drake 
Passage and Mediterranean Sea assessed by real-time quantitative PCR. Environ. Microbiol. 11: 397-408. doi:10.1111/ j.1462-2920.2008.01779.x

Rodríguez-Martínez, R., G. Rocap, G. Salazar, and R. Massana. 2013. Biogeography of the uncultured marine picoeukaryote MAST-4: Temperature-driven distribution patterns. ISME J. 7: 1531-1543. doi:10.1038/ismej.2013.53

Röttjers, L., and K. Faust. 2018. From hairballs to hypothesesbiological insights from microbial networks. FEMS Microbiol. Rev. 42: 761-780. doi:10.1093/femsre/fuy030

Schnetzer, A., S. D. Moorthi, P. D. Countway, R. J. Gast, I. C. Gilg, and D. A. Caron. 2011. Depth matters: Microbial eukaryote diversity and community structure in the eastern North Pacific revealed through environmental gene libraries. Deep-Sea Res. I Oceanogr. Res. Pap. 58: 16-26. doi:10. 1016/j.dsr.2010.10.003

Schoenle, A., M. Hohlfeld, K. Hermanns, F. Mahé, C. de Vargas, F. Nitsche, and H. Arndt. 2021. High and specific diversity of protists in the deep-sea basins dominated by diplonemids, kinetoplastids, ciliates and foraminiferans. Commun. Biol. 4: 1-10. doi:10.1038/s42003-02102012-5

Schön, M. E. Zlatogursky, V. V., Singh, R. P., others. 2021. Single cell genomics reveals plastid-lacking Picozoa are close relatives of red algae. bioRxiv 2021.04.14.439778. doi:10. 1101/2021.04.14.439778

Seenivasan, R., N. Sausen, L. K. Medlin, and M. Melkonian. 2013. Picomonas judraskeda gen. et sp. nov.: The first identified member of the Picozoa phylum nov., a widespread group of picoeukaryotes, formerly known as 'picobiliphytes'. PLoS One 8: e59565. doi:10.1371/journal. pone.0059565

Sekiguchi, H., M. Moriya, T. Nakayama, and I. Inouye. 2002. Vestigial chloroplasts in heterotrophic stramenopiles Pteridomonas danica and Ciliophrys infusionum (Dictyochophyceae). Protist 153: $157-167$. doi:10.1078/1434-4610-00094

Shalchian-Tabrizi, K., H. Kauserud, R. Massana, D. Klaveness, and K. S. Jakobsen. 2007. Analysis of environmental $18 \mathrm{~S}$ ribosomal RNA sequences reveals unknown diversity of the cosmopolitan phylum Telonemia. Protist 158: 173-180. doi:10.1016/j.protis.2006.10.003

Sherr, E. B., and B. F. Sherr. 2002. Significance of predation by protists in aquatic microbial food webs. Antonie Van Leeuwenhoek 81: 293-308. doi:10.1023/A:1020591307260

Shishkin, Y., D. Drachko, V. I. Klimov, and V. V. Zlatogursky. 2018. Yogsothoth knorrus gen. n., sp. n. and Y. carteri sp. n. (Yogsothothidae fam. n., Haptista, Centroplasthelida), with notes on evolution and systematics of centrohelids. Protist 169: 682-696. doi:10.1016/j. protis.2018.06.003

Šimek, K., and others. 2018. Bacterial prey food characteristics modulate community growth response of freshwater bacterivorous flagellates. Limnol. Oceanogr. 63: 484-502. doi:10.1002/lno.10759

Stoeck, T., D. Bass, M. Nebel, R. Christen, M. D. M. Jones, H. W. Breiner, and T. A. Richards. 2010. Multiple marker parallel tag environmental DNA sequencing reveals a highly complex eukaryotic community in marine anoxic water. Mol. Ecol. 19: 21-31. doi:10.1111/j.1365-294X. 2009.04480.x

Vaulot, D., S. Geisen, F. Mahé, and D. Bass. 2021. pr2-primers: An 18S rRNA primer database for protists. Mol. Ecol. Resour.: 1-12. doi:10.1111/1755-0998.13465

Watts, S. C., S. C. Ritchie, M. Inouye, and K. E. Holt. 2019. FastSpar: Rapid and scalable correlation estimation for compositional data. Bioinformatics 35: 1064-1066. doi:10. 1093/bioinformatics/bty734

Wickham, H., and others. 2019. Welcome to the Tidyverse. J. Open Source Softw. 4: 1686. doi:10.21105/joss.01686

Willis, A. D., and B. D. Martin. 2020. Estimating diversity in networked ecological communities. Biostatistics. doi:10. 1093/biostatistics/kxaa015

Zhu, F., R. Massana, F. Not, D. Marie, and D. Vaulot. 2005. Mapping of picoeucaryotes in marine ecosystems with quantitative PCR of the 18S rRNA gene. FEMS Microbiol. Ecol. 52: 79-92. doi:10.1016/j.femsec.2004.10.006

Živaljić, S., and others. 2018. Survival of marine heterotrophic flagellates isolated from the surface and the deep sea at high hydrostatic pressure: Literature review and own experiments. Deep-Sea Res. II Top. Stud. Oceanogr. 148: 251259. doi:10.1016/j.dsr2.2017.04.022

Zubkov, M. V., and G. A. Tarran. 2008. High bacterivory by the smallest phytoplankton in the North Atlantic Ocean. Nature 455: 224-226. doi:10.1038/nature07236

\section{Acknowledgments}

This research was supported by the Spanish Ministry of Science and Innovation projects Malaspina-2010 (CSD2008-00077), ALLFLAGS (CTM2016-75083-R) and the "Severo Ochoa Centre of Excellence" accreditation (CEX2019-000928-S), and the European Union project SINGEK (H2020-MSCA-ITN-2015-675752). AO was supported by a Spanish FPI grant. We thank all scientists and crew that participated in the Malaspina 2010 expedition, Ramiro Logares for the initial stages of the Micromap tool, and Ina M. Deutschmann for useful discussions on microbial networks. Bioinformatic analyses were performed at the Marbits platform (ICM-CSIC; https://marbits.icm.csic.es).

\section{Conflict of Interest}

None declared.

Submitted 12 July 2021

Revised 13 September 2021

Accepted 23 September 2021

Associate editor: Susanne Menden-Deuer 